\title{
Scanning Angle Raman Spectroscopy of Poly(3-hexylthiophene)- Based Films on Indium Tin Oxide, Gold, and Sapphire Surfaces
}

\author{
Matthew W. Meyer, ${ }^{\dagger \neq}$ Kelsey L. Larson, ${ }^{\dagger}$ Rakesh C. Mahadevapuram, ${ }^{\perp}$ Michael D. Lesoine, ${ }^{\dagger, \ddagger}$ \\ John A. Carr, ${ }^{\S}$ Sumit Chaudhary, ${ }^{\S, \perp}$ and Emily A. Smith ${ }^{*, \dagger, \ddagger}$ \\ ${ }^{\dagger}$ Ames Laboratory, U.S. Department of Energy, Ames, Iowa 50011-3111, United States \\ ${ }^{\ddagger}$ Department of Chemistry, ${ }^{\S}$ Department of Electrical and Computer Engineering, ${ }^{\perp}$ Department of Materials Science and \\ Engineering, Iowa State University, Ames, Iowa 50011-3111, United States
}

\section{Supporting Information}

ABSTRACT: Interest in realizing conjugated polymer-based films with controlled morphology for efficient electronic devices, including photovoltaics, requires a parallel effort to characterize these films. Scanning angle (SA) Raman spectroscopy is applied to measure poly(3-hexylthiophene) (P3HT):phenyl-C61-butyric acid methyl ester (PCBM)-blend morphology on sapphire, gold, and indium tin oxide interfaces, including functional organic photovoltaic devices. Nonresonant SA Raman spectra are collected in seconds with signal-to-noise ratios that exceed 80 , which is possible due to the reproducible SA signal enhancement. Raman spectra are collected as the incident angle of the $785 \mathrm{~nm}$ excitation laser is precisely varied upon a prism/sample interface from approximately 35 to $70^{\circ}$. The width of the $\sim 1447 \mathrm{~cm}^{-1}$ thiophene

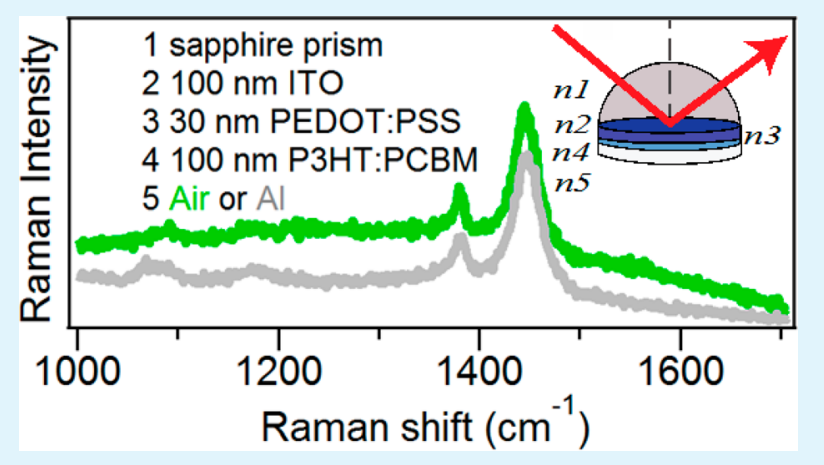
the importance of performing the spectroscopic mores the importance of performing the spectroscopic measurements on substrates and configurations used in the functioning devices, which is not a common practice. The experimental measurements are modeled with calculations of the interfacial mean square electric field to determine the distance dependence of the SA Raman signal. SA Raman spectroscopy is a versatile method applicable whenever the chemical composition, structure, and thickness of interfacial polymer layers need to be simultaneously measured.

KEYWORDS: conjugated polymer films, P3HT, phenyl-C61-butyric acid methyl ester, PCBM, thin film characterization, Raman spectroscopy

\section{INTRODUCTION}

Conjugated organic polymers and molecules with conducting properties are suitable for use in electronic devices, such as light-emitting diodes and photovoltaic devices. ${ }^{1}$ The performance of these organic-based devices highly depends on the ordering of molecules at the interface. For example, blending poly(3-hexylthiophene) ( $\mathrm{P} 3 \mathrm{HT}$ ) with the fullerene derivative phenyl-C61-butyric acid methyl ester (PCBM) forms a socalled "bulk heterojunction" system. ${ }^{2-5}$ The bulk heterojunction system takes advantage of phase segregation between P3HT donor and PCBM acceptor to facilitate charge separation and transport while still allowing for a film thickness sufficient for high optical absorption. Increased P3HT crystallization has been shown to increase the charge carrier mobility, optical absorption, and output efficiencies in organic photovoltaic devices. ${ }^{6,7}$ Therefore, understanding the molecular ordering of $\mathrm{P} 3 \mathrm{HT}$ is critical when making use of these thin films as active layers in various electronic devices.

The P3HT:PCBM morphology depends on several experimental factors including mixture composition, solvent, and processing steps. ${ }^{8}$ To date bulk heterojunctions have been fabricated by various processes including postproduction thermal annealing and slow growth solvent annealing. ${ }^{9}$ Slow growth solvent annealing involves storing the active layer in a restricted volume directly after spin coating to allow the solvent to naturally evaporate. ${ }^{10}$ It has been demonstrated that slow evaporation of the solvent leads to a dramatic improvement in the performance of the corresponding solar cells. ${ }^{11}$ The polymer thickness in functioning devices can range from 11 $\mathrm{nm}$ to a few hundred nanometers depending on the application requirements. ${ }^{12}$ Unfortunately, consistent control over donor:acceptor morphology at a variety of spatial scales, and thus efficient device performance, has proven to be a challenge. Over the past decade, incremental improvements in materials processing and the emergence of new materials have led to

Received: June 17, 2013

Accepted: August 16, 2013

Published: August 16, 2013 


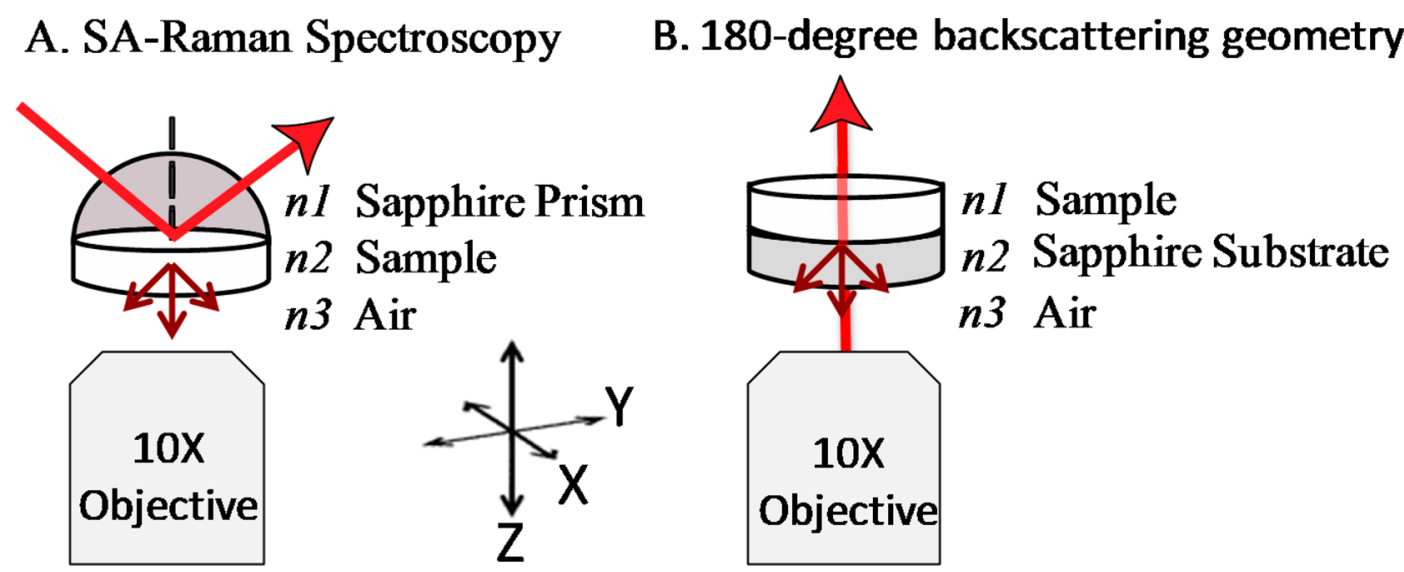

Figure 1. Schematic of the (A) scanning-angle Raman spectroscopy format used to measure P3HT-based films on sapphire, gold or ITO substrates, compared to (B) conventional, 180-degree backscattering Raman geometry. In the scanning angle Raman format, the incident angle of light relative to the axis shown by the dotted line is varied. The coordinate system is used throughout the discussion.

power conversion efficiencies of organic photovoltaic cells ranging from $\sim 4-9 \% .{ }^{13,14}$

Raman spectroscopy is a nondestructive vibrational technique that can be used to probe molecular information and P3HT film morphology. ${ }^{15}$ The $\sim 1447 \mathrm{~cm}^{-1} \mathrm{C}=\mathrm{C}$ thiophene ring stretch is sensitive to the extent of $\pi$-electron delocalization along the P3HT chain axis, and monitoring the Raman peak shape and position can give insight into morphology and crystallinity. ${ }^{16,17}$ Since P3HT's thiophene rings are more closely stacked with greater order when the polymer is highly crystalline, there is a narrowing of the $\sim 1447 \mathrm{~cm}^{-1}$ peak. Many studies report the full width at half-maximum (fwhm) of the $\sim 1447 \mathrm{~cm}^{-1}$ peak as a measure of P3HT crystallinity. ${ }^{16,18-20}$ High signal-to-noise ratio Raman spectra of nanometer thin films and accurate peak fitting algorithms are required to accurately correlate changes in Raman peak shape with polymer morphology.

Raman spectroscopy has been used to study P3HT and bulk heterojunction systems. ${ }^{10,21-24}$ Recently, Tosi and co-workers investigated how the $\mathrm{C}=\mathrm{C}$ Raman mode can be used as a tool to quantify the degree of molecular order of $\mathrm{P} 3 \mathrm{HT}$ in the P3HT:PCBM-blend films using resonance, preresonance and nonresonant conditions. ${ }^{20}$ The degree of molecular order measured by Raman spectroscopy correlated well with the performance of the corresponding solar cells. Resonance Raman imaging has also been applied to spatially map morphology dependent variations in P3HT:PCBM-blend films. 25

In this paper we show the application of scanning angle (SA) Raman spectroscopy for studying P3HT molecular order on various substrates relevant for organic electronic devices. The SA Raman configuration and conventional $180^{\circ}$ backscattering geometry are compared schematically in Figure 1. The SA Raman configuration generates a large mean square electric field (MSEF) within the polymer film at defined incident angles. This increases the generated Raman scattering compared to conventional Raman spectroscopy. SA Raman spectra of P3HT and P3HT:PCBM films have 80 to 230 signalto-noise ratios with 30 -s acquisition times and approximately 20 $\mathrm{mW}$ laser power at nonresonant conditions. The enhanced signal, without the use of resonant excitation, enables the characterization of changes in the $\mathrm{C}=\mathrm{C}$ thiophene peak shape in functional photovoltaic devices where the P3HT:PCBMblend is sandwiched between semitransparent layers. We show the degree of P3HT order varies for indium tin oxide, gold and sapphire substrates, and demonstrate the need to perform Raman measurements in the functional device to obtain relevant morphology information.

\section{EXPERIMENTAL SECTION}

2.1. Film Preparation. Thirty $\mathrm{mg} / \mathrm{mL}$ P3HT or $30 \mathrm{mg} / \mathrm{mL}$ PCBM (ChemSci technologies Inc., Belvidere, IL) were dissolved in 1,2-dichlorobenzene and stirred at $850 \mathrm{rpm}$ on a hot plate at a temperature of $60{ }^{\circ} \mathrm{C}$ for $24 \mathrm{~h}$ to make the active layer solution. All films were prepared in a glovebox filled with nitrogen. The solutions were filtered using a glass syringe to avoid contamination ${ }^{26}$ and a 0.2 $\mu \mathrm{m}$ filter (Millipore, Billerica, MA). After filtration the films were spin coated (Specialty Coating Systems Inc., Indianapolis, IN) onto a clean $25.4 \mathrm{~mm}$ sapphire disc (Meller optics, Providence, RI) or onto a sapphire disc with $50 \mathrm{~nm}$ gold (GWC technologies, Madison, WI or Center for Nanophase Materials Sciences, Oak Ridge National Laboratory). The spin-coating speeds were 600 and $500 \mathrm{rpm}$ for P3HT and PCBM, respectively. The remaining solvent was allowed to evaporate completely for $1 \mathrm{~h}$ after spin-coating. Subsequently, P3HT:PCBM (1:1 ratio, $8.5 \mathrm{mg} / \mathrm{mL}$ of each material in dichlorobenzene) were spin coated on sapphire or $50 \mathrm{~nm}$ gold substrates at $550 \mathrm{rpm}$. Again, the remaining solvent was allowed to evaporate completely for $1 \mathrm{~h}$.

A photovoltaic device was prepared by depositing approximately $100 \mathrm{~nm}$ of indium tin oxide (ITO) onto the sapphire substrate under RF plasma deposition conditions $\left(225^{\circ} \mathrm{C}, 90 \mathrm{~W}, 5 \mathrm{mT}\right)$. Poly $(3,4-$ ethylenedioxythio-phene):poly(styrenesulfonate) (PEDOT:PSS) (H.C. Stark, Newton, MA) was spin coated on the ITO side of the substrate at $4000 \mathrm{rpm}$ for $60 \mathrm{~s}$ with an approximate final thickness of $30 \mathrm{~nm}$. The P3HT:PCBM blend $(20 \mathrm{mg} / \mathrm{mL})$ in 1,2-dichlorobenzene solvent was then spin coated at $500 \mathrm{rpm}$ for $60 \mathrm{~s}$ and the remaining solvent was allowed to evaporate completely for $1 \mathrm{~h}$. The final P3HT:PCBM blend layer had a thickness of approximately $100 \mathrm{~nm}$ as previously measured by atomic force microscopy (AFM). Lastly where noted, an $\sim 150 \mathrm{~nm}$ aluminum cathode was thermally evaporated at a rate $<5 \AA / \mathrm{s}$ under a $1 \times 10^{-6} \mathrm{mBar}$ vacuum.

2.2. SA Raman Measurements. SA Raman spectroscopy experiments were performed using a previously described instrument. ${ }^{27}$ The instrument is capable of recording Raman spectra in the incident angle range of $28.00-74.00^{\circ}$ with a $0.05^{\circ}$ incident angle resolution. Raman spectra were collected in $1^{\circ}$ increments at the sapphire and ITO interfaces and between 0.05 (close to the plasmon waveguide resonance angle) to 1 degree at the gold interface. Incident excitation was from a 785-nm laser with $21 \mathrm{~mW}$ measured at the sample unless otherwise noted. The beam was $\sim 200 \mu \mathrm{m}$ in diameter at the sample interface. A $10 \times$ magnification objective was used to collect the Raman scattering. Immersion oil (Cargille Laboratories, Cedar 
Grove, NJ, $n=1.7800$ ) was used to ensure optical contact between a sapphire prism and the sapphire substrate on which the P3HT-based films were formed. A half-wave plate in the excitation path was used to control the polarization of the incident light at the sample interfaces. All SA Raman spectra were acquired with a 30-s acquisition time unless otherwise noted.

2.3. Absorption, Thickness, and AFM Film Characterization. Absorption spectra were recorded using an Agilent 8453 UV-visible spectrophotometer (Santa Clara, CA). The absorption spectra were recorded immediately following the Raman measurements. P3HT and P3HT:PCBM blend thicknesses on sapphire and gold were determined using a F20 thin film measurement system (Filmetrics, San Diego, CA) in reflective mode. The standard deviation was calculated from three replicate measurements from three different locations at or near the center of the film after absorption measurements were performed.

2.4. Mean Square Electric Field (MSEF) Calculations. A 3Dfinite-difference-time-domain (FDTD)-based simulation (EM Explorer, San Francisco, CA) was used to calculate the MSEF generated in the polymer layer and throughout the interface. The calculations assumed all individual layers had a constant index of refraction. The indices of refraction used for all calculations at $785 \mathrm{~nm}$ were: sapphire prism $\left(n_{\text {p-polarization }}=1.764, n_{s-\text { polarization }}=1.753\right)$, ITO $(n=1.858, k=$ $0.0116)$, P3HT ( $n=1.710, k=0.006)$, PEDOT:PSS $(n=1.44, k=$ $0.0544)$, P3HT:PCBM blend $(n=2.010, k=0.003)$, gold $(n=0.16, k$ $=4.71)$ and air $(n=1.000) .{ }^{28-34}$ A Yee cell size of $5 \mathrm{~nm}$ was used for all FDTD simulations. The angular resolution used for the calculations was $0.10^{\circ}$. The polymer and substrate layer thicknesses used in the FDTD calculations are provided in the figure legends.

Data Analysis. All data analysis was performed using the software IGOR Pro 6.1. Signal-to-noise ratios were calculated as the maximum of the $\sim 1447 \mathrm{~cm}^{-1}$ peak intensity after background subtraction divided by the standard deviation from the 1600 to $1625 \mathrm{~cm}^{-1}$ region of the spectrum where no analyte peaks were present. Peak fwhms were measured using the "Multipeak fitting 2" algorithm in IGOR Pro 6.1 using a Gaussian peak shape and linear baseline. The MSEF graphs were compiled in Matlab v.7.12.0 using a surface plot.

\section{RESULTS AND DISCUSSION}

SA Raman spectroscopy has previously been used to measure polystyrene films at sapphire, gold and silica interfaces. ${ }^{35-37}$ The goal of the current work is to apply SA Raman spectroscopy to measure thin film semiconducting polymers on sapphire, gold and ITO substrates, focusing on the variability of P3HT order at these interfaces. The P3HT, PCBM, and P3HT:PCBM Raman signal at each interface as a function of incident angle is first discussed, followed by a discussion of P3HT morphology.

SA Raman Spectra of P3HT, PCBM, and 1:1 P3HT:PCBM on Sapphire. The Raman spectra of P3HT, PCBM, and P3HT:PCBM on sapphire are shown in Figure 2 with the conventional $180^{\circ}$ backscattering configuration (Figure 2A) and the scanning-angle configuration (Figure $2 B)$. The Raman spectra plotted in Figure $2 \mathrm{~B}$ were collected at a $35.00^{\circ}$ incident angle, which is the angle that produced the largest Raman signal for all three samples. This is close to the sapphire/air critical angle of 34.59 degrees, as expected. With all experimental conditions the same except for the excitation geometry, it is evident that the SA Raman format enhances the Raman signal over the backscattering geometry. Compared to the backscattering geometry, the SA Raman spectra collected at $35.00^{\circ}$ have a $21 \times, 11 \times$, or $4 \times$ larger peak area for P3HT $(1447$ $\left.\mathrm{cm}^{-1}\right)$, PCBM $\left(1465 \mathrm{~cm}^{-1}\right)$, and P3HT:PCBM $\left(1447 \mathrm{~cm}^{-1}\right)$, respectively.

The absorption spectra shown in Figure 3 confirm that the $785 \mathrm{~nm}$ excitation wavelength corresponds to nonresonant

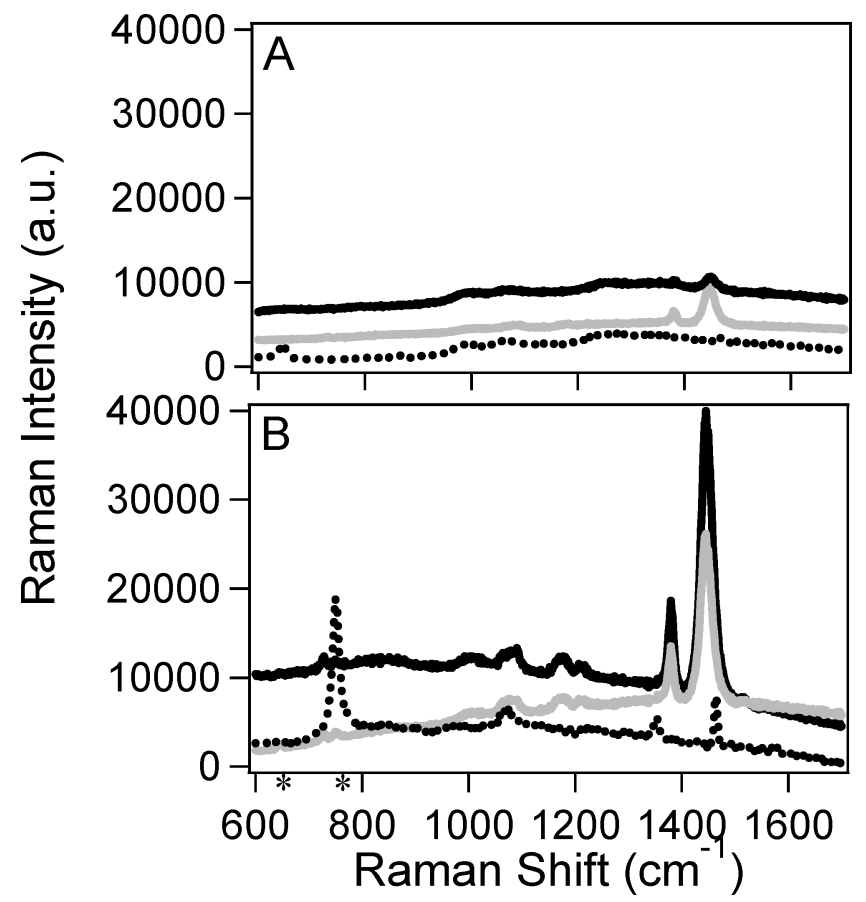

Figure 2. Raman spectra of P3HT (black), PCBM (dotted black), and a 1:1 mixture of P3HT:PCBM (gray) on sapphire. The Raman spectra were acquired with (A) $180^{\circ}$ backscattering Raman spectroscopy or with (B) SA Raman spectroscopy at an incident angle of $35.00^{\circ}$. The incident power at the sample (measured without a prism) was $21 \mathrm{~mW}$ for P3HT and P3HT:PCBM and $205 \mathrm{~mW}$ for PCBM. The peaks from the sapphire prism that appear in the SA Raman spectra are marked with asterisks $(*)$.

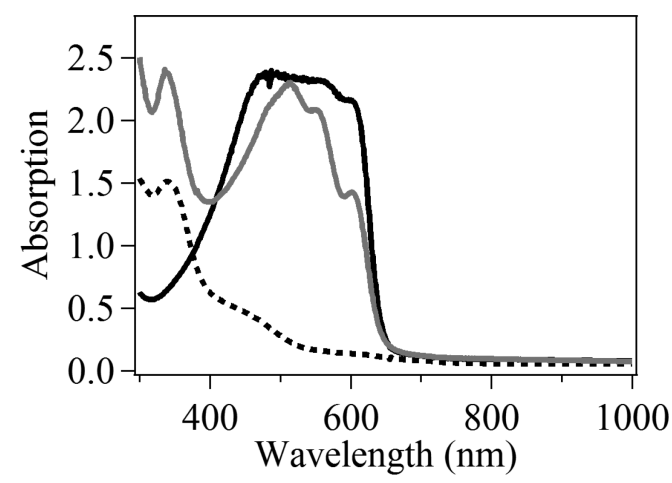

Figure 3. Absorption spectra of P3HT (black), PCBM (dotted black), and 1:1 P3HT:PCBM (gray) films spread on a sapphire substrate.

conditions for both P3HT and PCBM. To record a PCBM Raman spectrum, we required approximately an order of magnitude higher laser power compared to the $21 \mathrm{~mW}$ power that was used to collect the P3HT and P3HT:PCBM spectra. Therefore, the conditions used to acquire $\mathrm{P} 3 \mathrm{HT}$ spectra have minimal contribution from PCBM despite the similar Raman shifts of the main PCBM $\left(\sim 1465 \mathrm{~cm}^{-1}\right)$ and P3HT $(\sim 1447$ $\mathrm{cm}^{-1}$ ) peaks. All Raman peak locations and assignments are shown in the Supporting Information, Table S1.

The SA Raman spectra collected at selected incident angles for P3HT and PCBM on sapphire are shown in Figure 4. The signal for the P3HT:PCBM-blend is approximately half that of the pure $\mathrm{P} 3 \mathrm{HT}$ film, which is expected given the $1: 1$ composition of the P3HT:PCBM blend. Although the Raman signal of the P3HT:PCBM blend decreases with increasing 

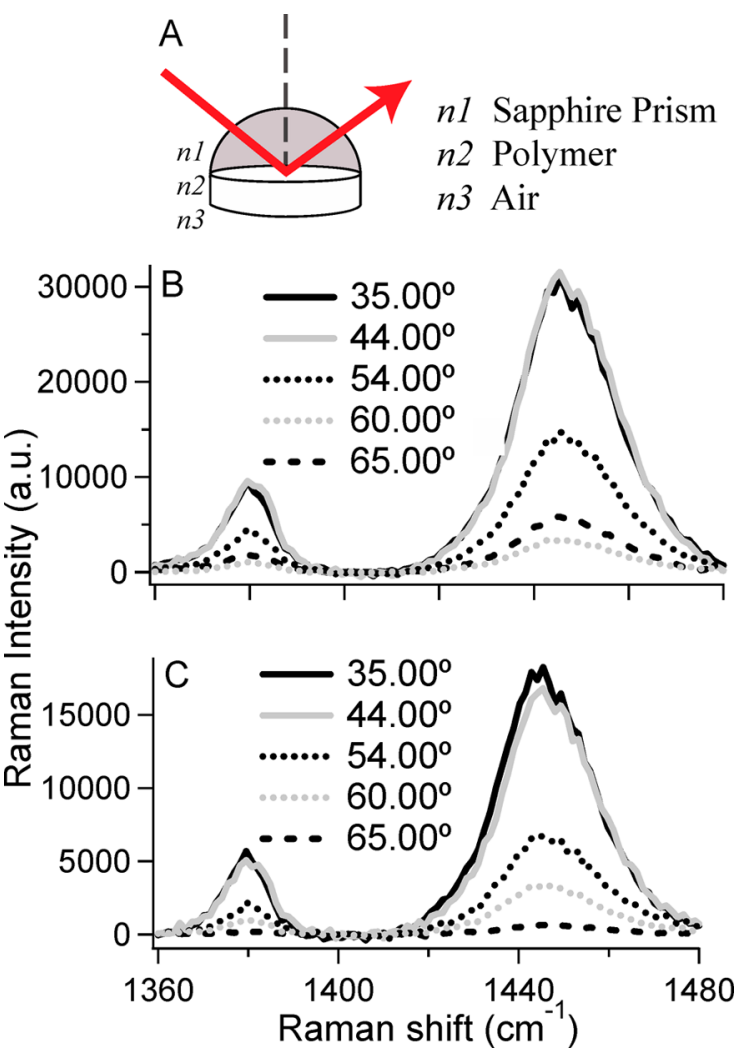

Figure 4. (A) Schematic of the SA Raman interface used to collect the data shown in B and C. SA Raman spectra at the indicated incident angles for (B) P3HT and (C) 1:1 P3HT:PCBM deposited on a sapphire substrate.

incident angle, the Raman signal for pure $\mathrm{P} 3 \mathrm{HT}$ decreases until the incident angle is scanned to approximately $60^{\circ}$, and then increases at larger incident angles.

MSEF calculations show the distance dependence in the $\mathrm{Z}$ direction (Figure 1) of the Raman signal, and aid in the interpretation of the Raman signal as the incident angle of excitation is varied (Figure 5). The expected Raman signal is proportional to the integrated MSEF across the entire polymer thickness (i.e., starting on the left of the plot and ending at the vertical white line). The MSEF plots shown in Figure 5 were calculated using the polymer thickness measured by AFM analysis on the samples used to generate the Raman data in Figure 4. The standard deviation for these AFM measurements was $100 \mathrm{~nm}$. Because the independent measurement of the film thickness by AFM is rather uncertain, only general trends between the experimental Raman data and the MSEF calculations will be considered.

Analysis of the MSEF within the polymer layer indicates that the Raman signal for the P3HT:PCBM films should decrease as the incident angle is scanned beyond 35 degrees, whereas the P3HT films should have a maximum near 35 degrees and a second maximum near 65 degrees (Figure 5). This agrees with the experimental Raman spectra as demonstrated by the subset of data shown in Figure 4. The MSEF plots reveal information regarding the spatial profile in the $Z$ direction: the experimental Raman signal is generated across the entire polymer film, but not uniformly throughout the polymer film at all incident angles. Polymer located in the region having the highest MSEF will contribute the most to the experimental Raman signal. Although the calculated MSEF is large in the bulk air layer (i.e.,
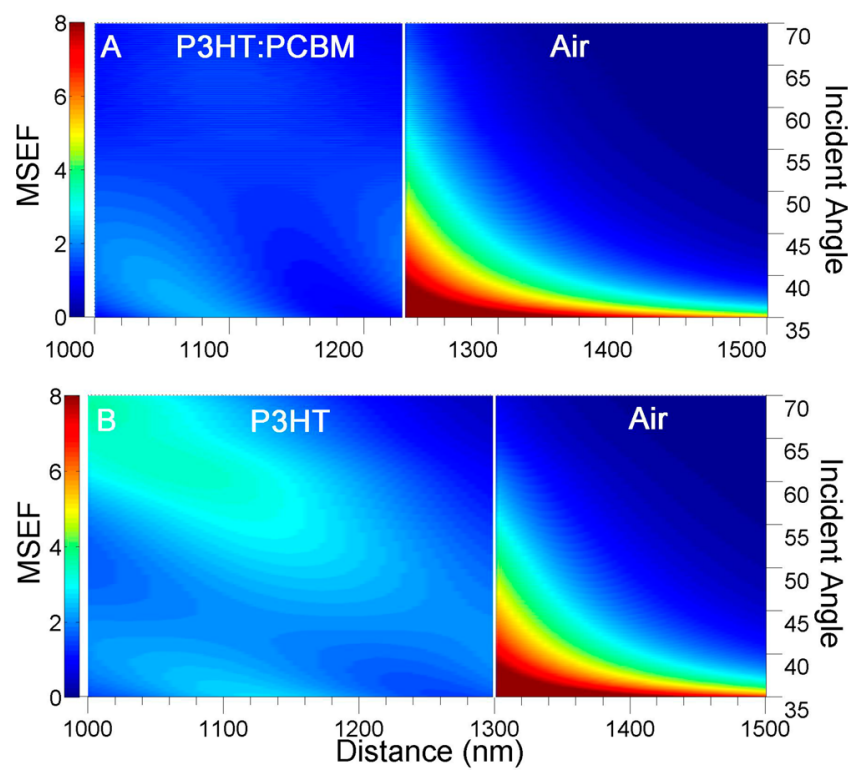

Figure 5. Calculated MSEF as a function of distance and incident angle for the interface: (A) $0-1000 \mathrm{~nm}$ sapphire/1000-1230 nm P3HT:PCBM/1230-6000 nm air, (B) 0-1000 nm sapphire/1000 to $1300 \mathrm{~nm} \mathrm{P3HT} / 1300$ to $6000 \mathrm{~nm}$ air. The MSEF in the sapphire layer $(0-1000 \mathrm{~nm})$ and the majority of the air layer (greater than $1500 \mathrm{~nm}$ ) are omitted for clarity. The calculated plots show the expected distance dependence of the experimental Raman signal in the $Z$ direction. The thicknesses of $230 \mathrm{~nm}$ P3HT:PCBM and $300 \mathrm{~nm}$ P3HT represent the sample thicknesses determined by AFM for the samples used to generate the data plotted in Figure 4.

to the right of the vertical white line), this will not affect the experimental Raman spectrum because the Raman signal of air is negligible. The MSEF's spatial profile throughout the interface depends on many factors including polymer thickness, incident angle, indices of refraction, and excitation wavelength.

In contrast to the spatial profile of the Raman signal in the $\mathrm{Z}$ direction, the spatial profile in the $X / Y$ direction (Figure 1 ) is controlled by the diameter of the laser beam at the sapphire/ sample interface. For the data presented herein the beam diameter is $200 \mu \mathrm{m}$, which is sufficiently large to average the signal from any lateral inhomogeneities in the polymer film.

SA Raman Spectra of P3HT and 1:1 P3HT:PCBM on Gold. Chemical and structural analysis of conducting polymers on metals has seen an increase in demand with the rise of light emitting and light harvesting devices such as nanoscale lasers, cavities and antennas. ${ }^{38-40}$ The MSEF calculations for P3HT or P3HT:PCBM films on a gold substrate (Figure 6) reveal a very different pattern compared to the MSEF calculated for a sapphire substrate (Figure 5). At specific incident angles there is a large MSEF increase in the polymer layer corresponding to the angles where plasmon waveguide resonances (PWRs) are excited. At these incident angles, the largest Raman scattering is predicted. The incident angle associated with exciting PWRs depends on properties of the gold film and similar properties discussed above for the sapphire substrate. In contrast to rough metal films, smooth metal films produce MSEFs that are reproducible and easily quantified. Importantly, when other experimental variables are known, the pattern of the Raman signal as a function of incident angle provides an accurate measure of polymer thickness. ${ }^{35}$

Panels A and B in Figure 6 show the calculated MSEF for a $512 \mathrm{~nm}$ 1:1 P3HT:PCBM film and a $700 \mathrm{~nm}$ P3HT film on a 


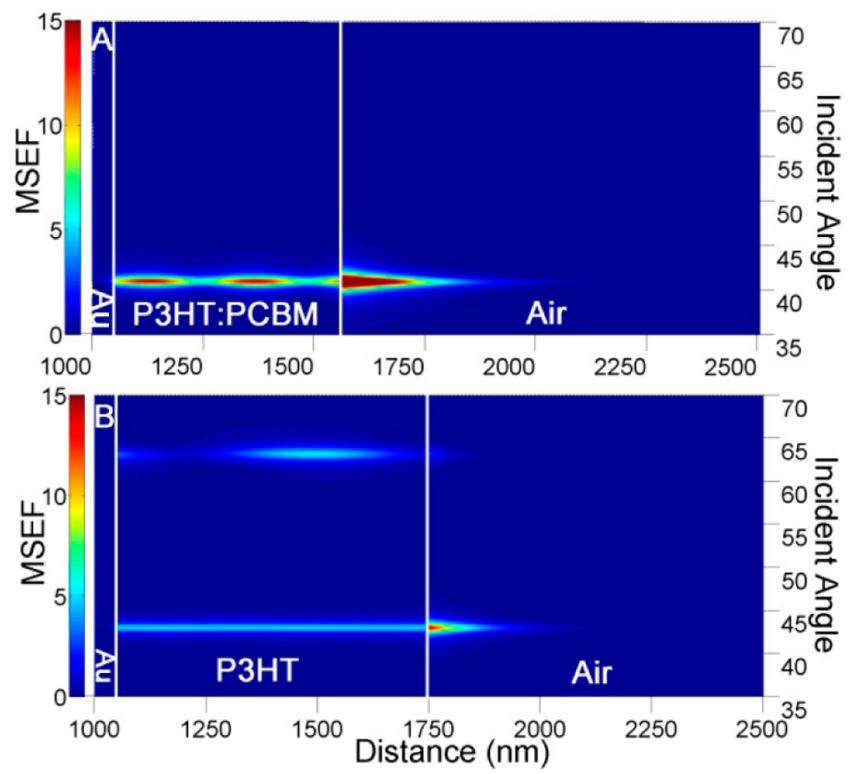

Figure 6. Calculated MSEF as a function of distance and incident angle for the interface: (A) $0-1000 \mathrm{~nm}$ sapphire/1000-1050 nm gold/1050 to $1562 \mathrm{~nm}$ P3HT:PCBM/1562-6000 nm air (B) 0-1000 $\mathrm{nm}$ sapphire/1000-1050 nm gold/1050-1750 nm P3HT/1750$6000 \mathrm{~nm}$ air. The MSEF in the sapphire layer $(0-1000 \mathrm{~nm})$ and the majority of the air layer (greater than $2500 \mathrm{~nm}$ ) are omitted for clarity. The calculated plots show the predicted incident angles that will generate the largest SA Raman scattering, which can be used to measure polymer thickness, and the distance dependence of the Raman signal in the $Z$ direction. The thicknesses $512 \mathrm{~nm}$ P3HT:PCBM and $700 \mathrm{~nm}$ P3HT provided the best fit to the experimental SA Raman data in Figure 7.

gold substrate. These polymer thicknesses best fit the SA Raman data represented in Figure 7 (only a subset of spectra are shown for clarity). Optical interferometry performed on the same polymer films generated thickness values of $570 \pm 50 \mathrm{~nm}$ and $830 \pm 40 \mathrm{~nm}$, respectively. Based on previously published work, there is an average $3 \%$ difference between polystyrene thickness measured by SA Raman spectroscopy and optical interferometry. ${ }^{35}$ It is reasonable that the lack of agreement between the two measurement techniques for the P3HT:PCBM film represents variability in roughness at the probed locations. A $10 \mu \mathrm{m} \times 10 \mu \mathrm{m}$ AFM image of the P3HT:PCBM film had a $z$-range roughness of $72 \mathrm{~nm}$ (see the Supporting Information, Figure S1), which supports this hypothesis. For a $43.00^{\circ}$ incident angle, the Raman signal generated in the $700 \mathrm{~nm}$ P3HT film is spatially uniform across the entire polymer thickness based on the MSEF plots, whereas a more complex spatial pattern in the $Z$ direction is expected for the Raman signal of the $512 \mathrm{~nm}$ P3HT:PCBM film at 40.90 degrees (Figure 6).

SA Raman Spectra of 1:1 P3HT:PCBM on Sapphire/ ITO/PEDOT:PSS. It is possible to measure the SA Raman spectrum of P3HT:PCBM films in functional organic photovoltaic devices. MSEF calculations of P3HT:PCBM on an sapphire/ITO/PEDOT:PSS interface were performed using ppolarized excitation (Figure $8 \mathrm{~A}$ ) or s-polarized excitation (Figure $8 \mathrm{~B}$ ). The MSEF distribution plot predicts $2.5 \times$ more Raman scattering will be generated within the P3HT:PCBM film with s-polarized excitation compared to p-polarized excitation at 60.00 degrees. In addition p-polarized excitation is predicted to generate significant Raman scattering within the
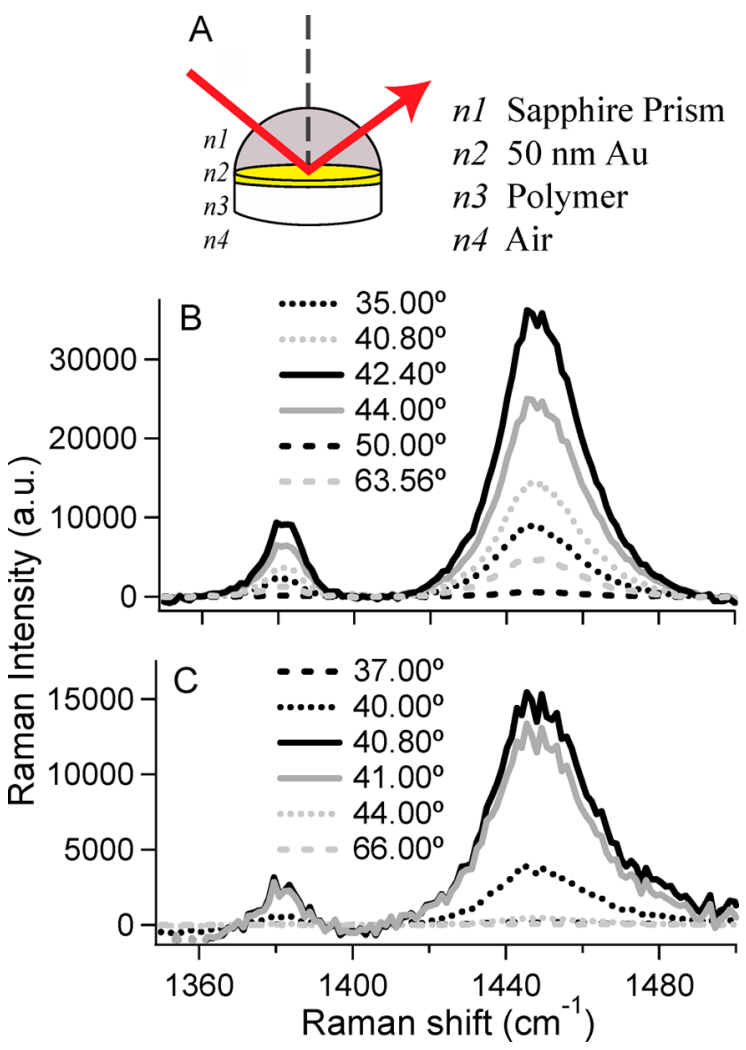

Figure 7. (A) Schematic of a sapphire/gold/polymer/air interface. Scanning angle Raman spectra at the indicated incident angles for (B) P3HT and (C) 1:1 P3HT:PCBM deposited on a gold substrate.

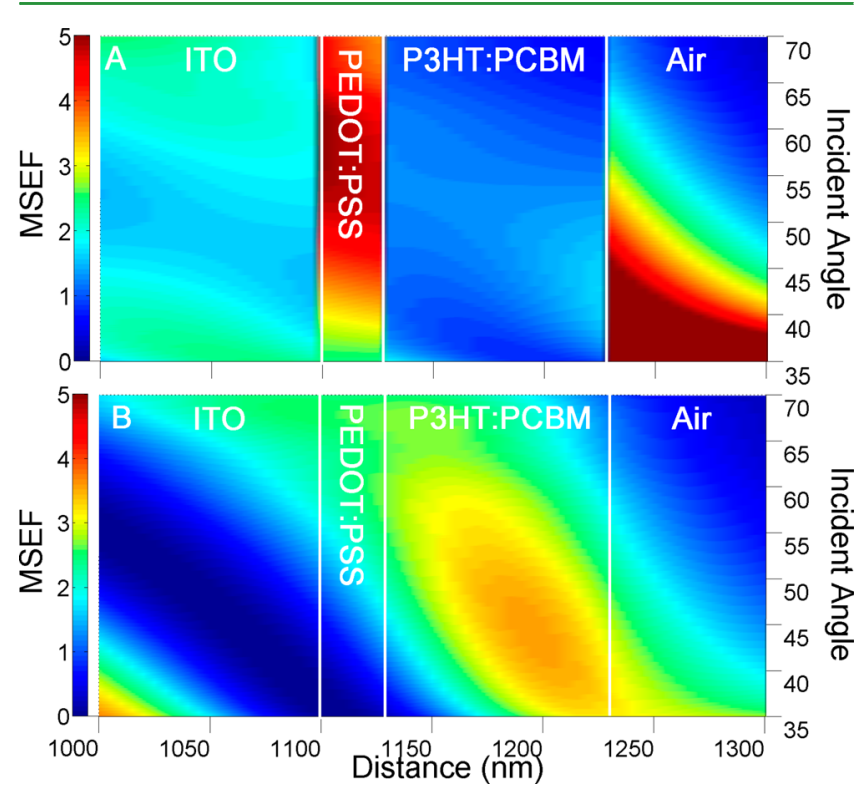

Figure 8. Calculated MSEF for the interface $0-1000 \mathrm{~nm}$ sapphire/ 1000-1100 nm ITO/1100-1130 nm PEDOT:PSS/1130-1230 nm P3HT:PCBM/1230-6000 nm air with (A) p- and (B) s-polarized excitation. The MSEF in the sapphire layer $(0-1000 \mathrm{~nm}$ ) and the majority of the air layer (greater than $1300 \mathrm{~nm}$ ) are omitted for clarity. The MSEF distribution plot predicts that more Raman scattering will be generated in the P3HT:PCBM film with s-polarized excitation while p-polarized excitation will generate strong, unwanted Raman scattering in the PEDOT:PSS film. 
$30 \mathrm{~nm}$ PEDOT:PSS film. PEDOT may spectrally interfere with the P3HT signal given the similar chemical functionalities in both polymers.

Another consideration when selecting excitation-polarization conditions using the SA Raman format is the orientation of the generated electric field at the interface. S-polarized excitation generates a MSEF component in the $\mathrm{Y}$ direction and $\mathrm{p}$ polarized excitation generates MSEF components in the $\mathrm{Z}$ and to a minor extent $\mathrm{X}$ directions. In the case of thiophene, both the $\mathrm{C}-\mathrm{C}$ and $\mathrm{C}=\mathrm{C}$ stretches are depolarized bands. ${ }^{41}$ With all of the above considerations, it is optimal to use s-polarized excitation for the experimental measurements on the sapphire/ ITO/PEDOT:PSS interface to maximize the P3HT contribution to the Raman spectrum.

Raman spectra collected at a $60.00^{\circ}$ incident angle are shown in Figure 9 for a sapphire/ITO/PEDOT:PSS/P3HT:PCBM

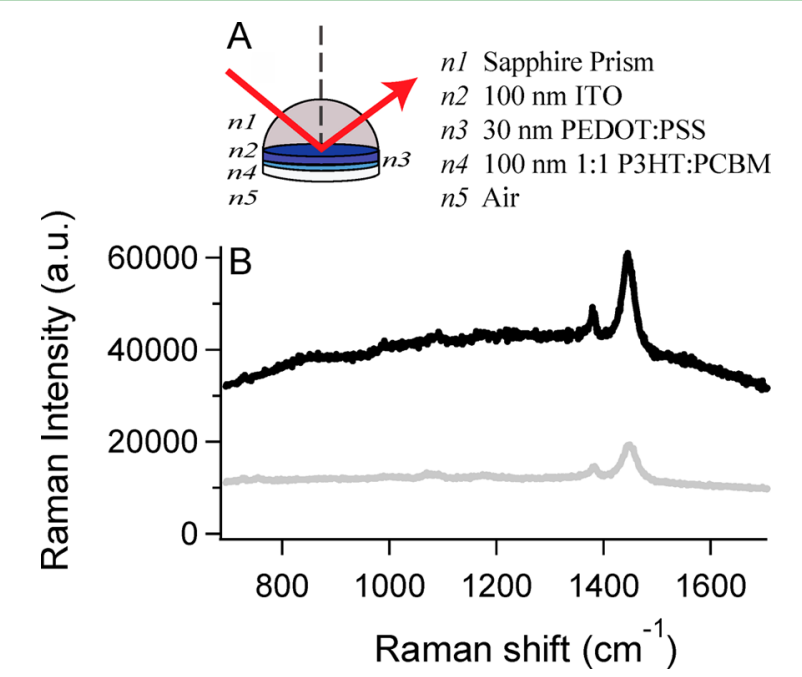

Figure 9. (A) Schematic of a sapphire/ITO/PEDOT:PSS/1:1 P3HT:PCBM/air interface. (B) Raman spectra of 1:1 P3HT:PCBM with $\mathrm{Al}$ coated over the polymer (gray) and without $\mathrm{Al}$ (black). The Raman spectra were collected at an incident angle of $60.00^{\circ}$ with spolarized excitation and $21 \mathrm{~mW}$ (black) or $40 \mathrm{~mW}$ (gray) laser power.

and sapphire/ITO/PEDOT:PSS/P3HT:PCBM/Al device, the latter represents a functional photovoltaic device. Since the aluminum layer is semitransparent, the laser power was increased approximately $2 \times$ to record the spectrum of this sample. Surprisingly, the aluminum layer does not significantly degrade the quality of the Raman spectrum, and reduces the spectral background compared to the sample without the aluminum layer. This indicates that the SA Raman measurements can be obtained on functional organic photovoltaic devices, which is beneficial to obtaining a direct correlation between polymer morphology and device performance.

Molecular Order of P3HT and 1:1 P3HT:PCBM Films. Addition of PCBM to the P3HT film has been shown to increase P3HT molecular disorder. ${ }^{8}$ The degree of P3HT molecular order in the P3HT:PCBM films is well correlated with the performance of the corresponding photovoltaic device. SA Raman spectroscopy is ideally suited to measure the effect of sapphire, gold or ITO/PEDOT:PSS substrates on P3HT molecular order using the same metrics described for Raman spectra collected with traditional illumination schemes. The fwhm of the $\sim 1447 \mathrm{~cm}^{-1}$ peak is expected to decrease, and device efficiency increase, with increasing $\mathrm{P} 3 \mathrm{HT}$ order or crystallinity. For all collected SA Raman spectra, the $\sim 1447$ $\mathrm{cm}^{-1} \mathrm{C}=\mathrm{C}$ stretching region of P3HT was fit to a Gaussian function. The use of a Gaussian function is suitable for solid samples when molecular motion is minimal. ${ }^{42}$ Example Gaussian fits to the Raman spectra are shown in the Supporting Information, Figure S2. Although there was slight asymmetry between the $\sim 1447 \mathrm{~cm}^{-1}$ Raman peaks in all spectra and the corresponding Gaussian fit, suggesting the existence of at least two overlapping peaks, fitting this spectral region to two peaks resulted in large uncertainties in the 6 required fit parameters. Given the small residual when using a Gaussian fit (see the Supporting Information, Figure S2), the $\sim 1447 \mathrm{~cm}^{-1} \mathrm{C}=\mathrm{C}$ stretching region was fit to single Gaussian function and the fwhm calculated from the fit for spectra collected at each interface and incident angle. There was no correlation between fwhm and incident angle, and the measurement reproducibility was excellent (see the Supporting Information, Figure S3), thus the average fwhm for all incident angles was calculated (Table 1).

Table 1. Average Raman Shift and FWHM of the $\mathrm{C}=\mathrm{C}$ Stretch and the Signal-to-Noise Ratio for the Spectrum Collected at the Indicated Incident Angle That Produced the Largest Raman Signal

\begin{tabular}{|c|c|c|c|}
\hline interface $^{a}$ & $\begin{array}{c}\mathrm{C}=\mathrm{C} \text { Raman } \\
\text { shift }\left(\mathrm{cm}^{-1}\right)\end{array}$ & $\begin{array}{c}\mathrm{C}=\mathrm{C} \text { Raman } \\
\text { peak fwhm } \\
\left(\mathrm{cm}^{-1}\right)\end{array}$ & $\begin{array}{l}\text { signal-to- } \\
\text { noise ratio } \\
\text { (incident } \\
\text { angle, deg) }\end{array}$ \\
\hline sapphire/P3HT & $1446.2 \pm 0.5$ & $26.0 \pm 0.2$ & $\begin{array}{l}200 \\
(35.00)\end{array}$ \\
\hline sapphire/P3HT:PCBM blend & $1446.1 \pm 0.3$ & $25.8 \pm 0.4$ & $\begin{array}{l}150 \\
(35.00)\end{array}$ \\
\hline sapphire/Au/P3HT & $1448.4 \pm 0.4$ & $26.0 \pm 0.2$ & $\begin{array}{l}230 \\
(43.00)\end{array}$ \\
\hline $\begin{array}{l}\text { sapphire/Au/P3HT:PCBM } \\
\text { blend }\end{array}$ & $1448.7 \pm 0.3$ & $29 \pm 1$ & $\begin{array}{l}80 \\
(41.60)\end{array}$ \\
\hline $\begin{array}{l}\text { sapphire/ITO/PEDOT:PSS/ } \\
\text { P3HT:PCBM blend } \\
\text { S-polarization }\end{array}$ & $1446.2 \pm 0.6$ & $27.8 \pm 0.8$ & $\begin{array}{l}150 \\
(60.0)\end{array}$ \\
\hline $\begin{array}{l}\text { sapphire/ITO/PEDOT:PSS/ } \\
\text { P3HT:PCBM blend/Al } \\
\text { S-polarization, } 40 \mathrm{~mW}\end{array}$ & $1450 \pm 2$ & $31 \pm 1$ & $\begin{array}{l}155 \\
(60.0)\end{array}$ \\
\hline
\end{tabular}

${ }^{a_{T}}$ The incident laser was p-polarized and $21 \mathrm{~mW}$ at the sample unless otherwise noted.

All of the samples included in this study used slow growth solvent annealing post processing. For the sapphire substrate, there is no evidence for an increase in P3HT disorder in the P3HT:PCBM blend. The fwhm of the $\mathrm{C}=\mathrm{C}$ peak is approximately $26 \mathrm{~cm}^{-1}$ with and without PCBM added to the P3HT. This indicates that PCBM does not increase P3HT disorder on the sapphire substrate when slow growth solvent annealing is used as a post processing step. This conclusion is supported by the absorption spectrum in Figure $2 .{ }^{22}$ On the gold interface, however, the fwhm increases from $26.2 \pm 0.2$ (P3HT) to $29 \pm 1$ (P3HT:PCBM) $\mathrm{cm}^{-1}$. This indicates P3HT order on a gold substrate decreases when PCBM is added. Tsoi et al. reported the fwhm for the $\mathrm{C}=\mathrm{C}$ stretch of a P3HT:PCBM film using $785 \mathrm{~nm}$ excitation before and after annealing the sample to increase P3HT order. ${ }^{20}$ Before annealing the fwhm was $39 \mathrm{~cm}^{-1}$ and after annealing the fwhm decreased to $26 \mathrm{~cm}^{-1}$. This supports the conclusion reported herein that $\mathrm{P} 3 \mathrm{HT}$ in the $\mathrm{P} 3 \mathrm{HT}: \mathrm{PCBM}$ blend on the gold substrate has increased disorder, however not to the same 
extent expected for a sample that had no post processing treatments. Comparing the two films on the sapphire/ITO/ PEDOT:PSS substrate, the additional processing required to form the aluminum electrode on the polymer films increases P3HT disorder compared to the film without the aluminum film.

These conclusions were confirmed with two additional experiments: AFM on selected samples and resonant Raman spectroscopy using traditional $180^{\circ}$ backscattering illumination. Raman spectroscopy with $488 \mathrm{~nm}$ excitation corresponds to resonance Raman conditions (Figure 3). Resonance Raman spectra showed a $\sim 1447 \mathrm{~cm}^{-1}$ fwhm ratio between the P3HT:PCBM and P3HT samples of $1.06 \pm 0.03$ with a sapphire substrate and $1.15 \pm 0.03$ with a gold substrate (see the Supporting Information, Figure S4 and Table S2). This compares well to the ratio collected using SA Raman spectroscopy $(0.99 \pm 0.03$ for sapphire and $1.12 \pm 0.04$ for gold); and validates the SA Raman measurements showing relative disorder for the P3HT:PCBM film on a gold substrate. Resonance conditions are not required to measure P3HT order using SA Raman spectroscopy.

AFM images were collected to measure the surface roughness in a P3HT film on sapphire and a P3HT:PCBM film on gold. The amount of P3HT ordering can be determined by the surface roughness. ${ }^{43}$ The P3HT film on sapphire had an RMS roughness of $19 \mathrm{~nm}$, and the P3HT:PCBM blend on gold had an RMS roughness of 8.3 (see the Supporting Information, Figure S1). When the P3HT order increases upon solvent vapor annealing, Miller et al. report the AFM images transformed from featureless to show 10 to $20 \mathrm{~nm}$ surface clusters; and the device with the higher surface roughness exhibited higher device efficiencies. ${ }^{19}$ Therefore, it can be concluded from the AFM images that there is more ordering of P3HT on sapphire than exhibited by the polymer in the P3HT:PCBM blend on gold. This is consistent with the conclusion drawn from analyses of the fwhm of the $\sim 1447$ $\mathrm{cm}^{-1}$ peaks. SA Raman spectroscopy indicated the P3HT exhibited relative order on sapphire and relative disorder in the P3HT:PCBM blend on the gold film.

The signal-to-noise ratio for the spectrum that produced the largest Raman signal was calculated at each interface (Table 1). All spectra were collected with a $30 \mathrm{~s}$ acquisition time, resulting in signal-to-noise ratios of at least 80 . Significantly shorter acquisition times can be used while still collecting spectra of sufficient quality for quantitative analysis. It should be noted that the signal-to-noise ratio can decrease significantly if the Raman spectra are not collected at the optimum incident angle, which highlights the importance of either scanning the range of incident angles or using MSEF calculations to determine optimal angles for data collection.

\section{CONCLUSIONS}

SA Raman spectroscopy is a powerful technique for studying molecular order in conjugated polymer and blend films. By utilizing SA Raman spectroscopy one can measure enhanced Raman signals, film thickness, molecular order, and one can determine where the Raman scattering is generated within the polymer film when combined with MSEF calculations. This technique is currently being used to measure other semiconducting photovoltaic materials such as PCDTBT (poly $[\mathrm{N}$ $9^{\prime}$-heptadecanyl-2,7-carbazole-alt-5,5-(4',7' -di-2-thienyl$2^{\prime}, 1^{\prime}, 3^{\prime}$-benzothiadiazole) $\left.]\right)$; and the SA Raman technique is being expanded to other excitation wavelengths.

\section{ASSOCIATED CONTENT}

\section{Supporting Information}

Raman peak assignments, Gaussian fit curves and fit parameters to Raman peaks using 488 and $785 \mathrm{~nm}$ excitation wavelengths, replicate SA Raman spectra, and AFM images. This material is available free of charge via the Internet at http://pubs.acs.org

\section{AUTHOR INFORMATION}

\section{Corresponding Author}

*E-mail: esmith1@iastate.edu.

\section{Notes}

The authors declare no competing financial interest.

\section{ACKNOWLEDGMENTS}

The SA Raman studies (M.W.M., M.D.L., E.A.S.) were supported by the U.S. Department of Energy, Office of Basic Energy Sciences, Division of Chemical Sciences, Geosciences, and Biosciences through the Ames Laboratory. The Ames Laboratory is operated for the U.S. Department of Energy by Iowa State University under Contract DE-AC02-07CH11358. K.L.L. was supported by the U.S. Department of Energy, Science Undergraduate Laboratory Internship program. Film preparation and device fabrication (R.C.M., J.A.C., S.C.) were supported by a National Science Foundation Award ECCS1055930. The authors thank the Iowa State University Microelectronics Research Center for use of their Filmetrics instrument and Mr. Jonathan Bobbit for helpful discussion. A portion of this research was conducted at the Center for Nanophase Materials Sciences, which is sponsored at Oak Ridge National Laboratory by the Scientific User Facilities Division, Office of Basic Energy Sciences, U.S. Department of Energy.

\section{REFERENCES}

(1) Sirringhaus, H. Nat. Mater. 2003, 2, 641-642.

(2) Halls, J. J. M.; Walsh, C. A.; Greenham, N. C.; Marseglia, E. A.; Friend, R. H.; Moratti, S. C.; Holmes, A. B. Nature 1995, 376, 498500.

(3) Yu, G.; Gao, J.; Hummelen, J. C.; F. Wudl, F.; Heeger, A. J. Science 1995, 270, 1789-1791.

(4) Li, Y. Acc. Chem. Res. 2012, 45, 723-733.

(5) Ayzner, A. L.; Tassone, C. J.; Tolbert, S. H.; Schwartz, B. J. J. Phys. Chem. C 2009, 113, 20050-20060.

(6) Sirringhaus, H.; Brown, P. J.; Friend, R. H.; Nielsen, M. M.; Bechgaard, K.; Langeveld-Voss, B. M. W.; Spiering, A. J. H.; Janssen, R. A. J.; Meijer, E. W.; Herwig, P.; De Leeuw, D. M. Nature 1999, 401, $685-688$.

(7) Sariciftci, N. S.; Smilowitz, L.; Heeger, A. J.; Wudl, F. Science 1992, 258, 1474-1476.

(8) Günes, S.; Neugebauer, H.; Sariciftci, N. S. Chem. Rev. 2007, 107, 1324-1338.

(9) Carr, J. A.; Chen, Y.; Elshobaki, M.; Mahadevapuram, R. C.; Chaudhary, S. Nanomater. Energy 2012, 1, 18-26.

(10) Nalwa, K. S.; Mahadevapuram, R. C.; Chaudhary, S. Appl. Phys. Lett. 2011, 98, 093306.

(11) Li, G.; Shrotriya, V.; Huang, J.; Yao, Y.; Moriarty, T.; Emery, K.; Yang, Y. Nat. Mater. 2005, 4, 864-868.

(12) Jia, H. P.; Gowrisanker, S.; Pant, G. K.; Wallace, R. M.; Gnade, B. E. J. Vacuum Science \& Technology A 2006, 24, 1228-1232.

(13) He, Z.; Zhong, C.; Huang, X.; Wong, W. Y.; Wu, H.; Chen, L.; Su, S.; Cao, Y. Adv. Mater. 2011, 23, 4636-4643.

(14) He, Z.; Zhong, C.; Su, S.; Xu, M.; Wu, H.; Cao, Y. Nat. Photonics 2012, 6, 591-595.

(15) Raman Spectroscopy for Chemical Analysis; McCreery, R. L., Ed.; Wiley-Interscience: New York, 2000; Vol. 157. 
(16) Wang, X.; Zhang, D.; Braun, K.; Egelhaaf, H. J.; Brabec, C. J.; Meixner, A. J. Adv. Funct. Mater. 2010, 20, 492-499.

(17) Carach, C.; Gordon, M. J. J. Phys. Chem. B 2013, 117, 19501957.

(18) Yun, J. J.; Peet, J.; Cho, N. S.; Bazan, G. C.; Lee, S. J.; Moskovits, M. Appl. Phys. Lett. 2008, 92, 251912.

(19) Miller, S.; Fanchini, G.; Lin, Y.-Y.; Li, C.; Chen, C.-W.; Su, W.F.; Chhowallaa, M. J. Mater. Chem. 2008, 18, 206-312.

(20) Tsoi, W. C.; James, D. T.; Kim, J. S.; Nicholson, P. G.; Murphy, C. E.; Bradley, D. D. C.; Nelson, J.; Kim, J. S. J. Am. Chem. Soc. 2011, 133, 9834-9843.

(21) Klimov, E.; Li, W.; Yang, X.; Hoffmann, G. G.; Loos, J. Macromolecules 2006, 39, 4493-4496.

(22) Janssen, G.; Aguirre, A.; Goovaerts, E.; Vanlaeke, P.; Poortmans, J.; Manca, J. Eur. Phys. J.: Appl. Phys. 2007, 37, 287-290.

(23) Malgas, G. F.; Arendse, C. J.; Mavundla, S.; Cummings, F. R. J. Mater. Sci. 2008, 43, 5599-5604.

(24) Carach, C.; Riisness, I.; Gordon, M. J. Appl. Phys. Lett. 2012, 101, 083302.

(25) Gao, Y. Q.; Grey, J. K. J. Am. Chem. Soc. 2009, 131, 9654-9662.

(26) Carr, J. A.; Nalwa, K. S.; Mahadevapuram, R.; Chen, Y.; Anderegg, J.; Chaudhary, S. ACS Appl. Mater. Interfaces 2012, 4, 28312835.

(27) McKee, K. J.; Smith, E. A. Rev. Sci. Instrum. 2010, 81, 043106043106-6.

(28) Handbook of Optical Constants of Solids III; Tropf, W. J.; Thomas, M. E., Eds.; Academic Press: San Diego, 1998.

(29) Malitson, I. H.; Murphy, J. F. V.; Rodney, W. S. J. Opt. Soc. Am. 1958, 48, 72-73.

(30) Laux, S.; Kaiser, N.; Zöller, A.; Götzelmann, R.; Lauth, H.; Bernitzki, H. Thin Solid Films 1998, 335, 1-5.

(31) Ordal, M. A.; Long, L. L.; Bell, R. J.; Bell, S. E.; Bell, R. R.; Alexander, J. R. W.; Ward, C. A. Appl. Opt. 1983, 22, 1099-1119.

(32) Schulz, L. G.; Tangherlini, F. R. J. Opt. Soc. Am. 1954, 44, 362367.

(33) Gevaerts, V. S.; Koster, L. J. A.; Wienk, M. W.; Janssen, R. A. J. ACS Appl. Mater. Interfaces 2011, 3, 3252-3255.

(34) Moulé, A. J.; Meerholz, K. Appl. Phys. Lett. 2007, 91, 061901.

(35) Meyer, M. W.; McKee, K. J.; Nguyen, V. H. T.; Smith, E. A. J.

Phys. Chem. C 2012, 116, 24987-24992.

(36) Meyer, M. W.; Nguyen, V. H. T.; Smith, E. A. Vib. Spectrosc. 2012, 65, 94-100.

(37) McKee, K. J.; Meyer, M. W.; Smith, E. A. Anal. Chem. 2012, 84, 9049-9055.

(38) Lu, X.; Zhang, W.; Wang, C.; Wen, T.-C.; Wei, Y. Prog. Polym. Sci. 2011, 36, 671-712.

(39) Terzi, F.; Pasquali, L.; Seeber, R. Anal. Bioanal. Chem. 2013, 405, 1513-1535.

(40) Guimard, N. K.; Gomez, N.; Schmidt, C. E. Prog. Polym. Sci. 2007, 32, 876-921.

(41) Wu, X.-F.; Xuming Zheng, X.; Wang, H.-G.; Zhao, Y.-Y.; Guan, X.; Phillips, D. L.; Chen, X.; Fang, W. J. Chem. Phys. 2010, 133, 134507.

(42) Bradley, M. S.; Krech, J. H. J. Phys. Chem. 1992, 96, 75-79.

(43) Zhao, G.; He, Y.; Li, Y. Adv. Mater. 2010, 22, 4355-4358. 